\title{
Interventional Pain Treatment Using Nerve Block: Usefulness and Perspectives
}

\author{
Kiyoshige Ohseto and Hiroyuki Uchino
}

\subsection{Introduction}

Here, we introduce diagnostic nerve block, which is expected to become an important technique in the future, and we also describe its usefulness and perspectives. The goal of interventional treatment using nerve block is to make an accurate diagnosis of the pain and also to perform interventional therapy, such as injection of the appropriate drug in the vicinity of the nerves causing the pain, to achieve prompt pain relief. For this purpose, accurate identification of the damaged site and nerves is required. The nerve provocative test is always performed first to identify the damaged site. Second, the extent of sensory disturbance is estimated, i.e., paresthesia, from the neurological data. Moreover, if sensation in the region of pain is normal, the affected nerve is speculated based on the dermatome. On the other hand, if identification by the dermatome is difficult and the patient feels only motion pain, identification of the points of tenderness is important. The nerve causing the pain is sometimes located under the site of tenderness. First, to speculate the site of and the specific nerves causing the pain, a radiologist will sometimes be consulted for assistance in the interpretation of various diagnostic images, such as X-ray, magnetic resonance, computerized tomography, and ultrasound images. Second, a nerve block is performed to the nerve or joint site that was identified as being responsible for the pain, under fluoroscopy-guided method or ultrasound-guided method. The main aim of nerve block is to induce the same pain through the same nerves by drug injection through the block needle and then to check the disappearance of the pain by the injection of local anesthetics. This technique enables the clinician to make a functional diagnosis. The procedures of the neuroimaging and nerve block under X-ray fluoroscopy or ultrasound guidance described in this book enable neurofunctional identification of the nerves causing the pain, which has been difficult until now. Furthermore, if effective interventional treatment is performed for the targeted nerves or sites, long-term effects can be achieved. We would like all pain clinicians to become experts in the nerve block technique, by learning the procedures of each step, including diagnosis of the pain and estimation of the temporary effect of the nerve block, and then proceed to perform the interventional pain treatment to achieve favorable effects.

It is sometimes difficult to accurately characterize the pain using only pathological and morphological approaches. In such cases, functional diagnosis based on a nerve block is considered to play an important role in identifying the nerve causing the pain and its site, which could lead to a definitive diagnosis. If a diagnostic nerve block is applied for the treatment of pain that has been difficult to treat, this should lead to prompt pain amelioration.

The purpose of this book is also to assist in performing preventive analgesia and therapeutic nerve block effectively and safely. Furthermore, the most important point of this book is to open and establish new avenues for the concept of nerve block, through the introduction of accurate and safe procedures of the diverse types of diagnostic nerve block.

We sincerely hope that this book will contribute toward providing strategies of pain treatment to medical staff and patients who are interested in pain management.

\subsection{Postscripts}

The Japanese references are listed at the end of the Japanese (in Japanese) and relevant articles. We would like you to refer to the figures and pictures in the Japanese references.

K. Ohseto $(\bowtie) \cdot H$. Uchino

Department of Anesthesiology, Tokyo Medical University,

Tokyo, Japan

e-mail: ohseto@tokyo-med.ac.jp 